\title{
Meteorological information in GPS-RO reflected signals
}

\author{
K. Boniface ${ }^{1}$, J. M. Aparicio ${ }^{1}$, and E. Cardellach $^{2}$ \\ ${ }^{1}$ Data Assimilation and Satellite Meteorology, Environment Canada, Dorval, QC, Canada \\ ${ }^{2}$ Institute of Space Sciences, ICE-CSIC/IEEC, Barcelona, Spain
}

Received: 27 January 2011 - Published in Atmos. Meas. Tech. Discuss.: 24 February 2011

Revised: 18 June 2011 - Accepted: 7 July 2011 - Published: 18 July 2011

\begin{abstract}
Vertical profiles of the atmosphere can be obtained globally with the radio-occultation technique. However, the lowest layers of the atmosphere are less accurately extracted. A good description of these layers is important for the good performance of Numerical Weather Prediction (NWP) systems, and an improvement of the observational data available for the low troposphere would thus be of great interest for data assimilation. We outline here how supplemental meteorological information close to the surface can be extracted whenever reflected signals are available. We separate the reflected signal through a radioholographic filter, and we interpret it with a ray tracing procedure, analyzing the trajectories of the electromagnetic waves over a 3-D field of refractive index. A perturbation approach is then used to perform an inversion, identifying the relevant contribution of the lowest layers of the atmosphere to the properties of the reflected signal, and extracting some supplemental information to the solution of the inversion of the direct propagation signals. It is found that there is a significant amount of useful information in the reflected signal, which is sufficient to extract a stand-alone profile of the low atmosphere, with a precision of approximately $0.1 \%$. The methodology is applied to one reflection case.
\end{abstract}

\section{Introduction}

The measurement and interpretation of GPS radio occultation signals (GPS-RO) that have suffered only atmospheric refraction during their propagation is well established. This includes their description as propagating waves, and therefore the presence of diffraction. The analysis of such signals, and in particular of radio occultation data is of great interest

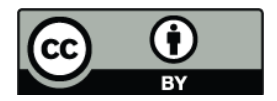

Correspondence to: $\mathrm{K}$. Boniface (karen.boniface@ec.gc.ca) for planetary atmospheres (Fjeldbo et al., 1971), Earth's atmospheric experimental and theoretical studies (Kursinski et al., 1996; Sokolovskiy, 1990; Gorbunov, 1988; Gurvich et al., 1982) and ionospheric studies (Raymund et al., 1990; Hajj et al., 1994). Indeed, beyond the geometric delay due to the finite speed of light, the delay in excess of this quantity contains information about the amount and distribution of matter encountered during the propagation, such as dry air and moisture in the atmosphere, and their gradients.

Over the last decades a number of studies have estimated the geophysical content of Global Positionning System (GPS) signals reflected off the surface of the Earth (Beyerle et al., 2002; Cardellach et al., 2008). To the presence of refraction, and eventually diffraction, this adds reflection. We will hereafter name as direct, the signals whose propagation is determined mainly by refraction phenomena, and eventually diffraction. We will instead name as reflected, the signals whose propagation involves critically some reflection at the surface, therefore introducing a qualitative difference with respect to the direct signals. Reflected signals are of course subject to refraction as well.

Reflected signals probe the entire atmosphere, down to the surface, which leaves an atmospheric signature in the received signal. In principle, the reflection itself may as well leave a signature in the signal, therefore obscuring the interpretation of the collected signal exclusively in terms of atmospheric properties. We have then limited here to the specific case where the signal bounces over the ocean, which is at a known altitude. At incidence angles closer to normal, the surface roughness, which is essentially determined by the presence of waves, is several times larger than the signal wavelength, and this can easily produce decoherence in the GPS signal. Coherence, however, is critical for all phasebased analyses of GPS signals, including GPS-RO. However, at very small incidence angles, the vertical scale that determines if close propagation paths are coherent is the Fresnel diameter of the propagation beam, which for paths near the

Published by Copernicus Publications on behalf of the European Geosciences Union. 
surface is of several hundred meters. Roughness and surface waves are much smaller than this. Therefore, at these incidence angles, they would not cause decoherence of the reflected signal.

Our attention is particularly dedicated to an improved description of the lowest layers of the atmosphere, were the inversion of direct GPS-RO signals is less accurate and subject to bias (Ao et al., 2003; Sokolovskiy et al., 2009). The refractivity bias in GPS occultation retrievals is related to the presence of strong vertical gradients of refractivity, mostly between the surface and the boundary layer. These strong gradients may cause superrefraction, or bend the signal outside the orbital segment where the receiver is active. In either case, there is a lack of information concerning these layers.

The main objective of the study is to explore the potential of GPS-RO signals that rebound off the ocean surface, and to determine whether there is geophysical information that can be accessed and be potentially useful to supplement the information extracted from the direct propagation channel. Reflected signals propagate following different geometries than the direct signals, and traverse atmospheric layers at different incidence angles. This may allow them to traverse layers that are superrefractive at lower incidence. There is therefore a potential to extract additional information with respect to the direct signals. Given that information describing the low troposphere is the most difficult to extract with direct propagation GPS-RO, and that information concerning these layers is the most valuable, we consider this potential worth exploring.

The value of GPS-RO data has otherwise already been analyzed in numerous global data assimilation experiments, and its geophysical data succesfully extracted from the analysis of direct propagation paths. These data are routinely used in operational Numerical Weather Prediction (NWP) systems (Healy, 2008; Cucurull et al., 2006; Aparicio and Deblonde, 2008; Rennie, 2010; Poli, 2008).

We will first use a radio-holographic technique to identify the propagation paths. Reflected signals present in GNSS occultation data present different frequency spectra than the direct signals (Beyerle et al., 2002; Cardellach et al., 2008). This allows a separation of both, and further independent treatment as data sources. For the interpretation, we use a numerical model based on ray tracing, where propagation trajectories of electromagnetic waves are evaluated over a given (a priori) 3-D field of refractive index. Supplemental information is also evaluated during the raytracing to allow a perturbation analysis. This latter describes the dependence of the raytracing result with respect to variations of the apriori field. Since the raytracing operates in a multidimensional vector space of solution profiles, we also illustrate the inversion procedure with a simpler case where this vector space is 1-D. The perturbation analysis allows a least-squares fit to the observed reflected data, which retrieves a correction to the apriori refractivity field.

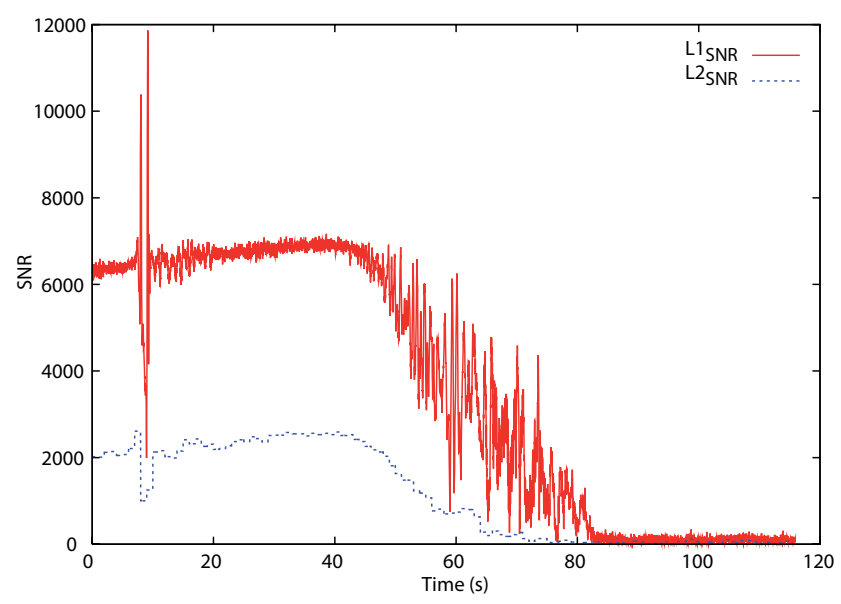

Fig. 1. Signal to Noise Ratio (SNR) on the L1 and L2 channels. SNR are given in arbitrary receiver units. After about 80 s the power is not evidently above the noise floor.

\section{Radio-holographic analysis to separate direct and reflected signals}

One possible procedure for the separation of the direct and reflected signals is a radio holographic analysis (Hocke et al., 1999). Signal to noise ratios (SNR), a measure of signal amplitude, hereafter expressed as $A(t)$, and carrier phase measurements $\varphi(t)$ have been recorded at a number of sampling instants $t$. As an example, we show in Fig. 1, the SNR for both carrier frequencies L1 and L2 during a setting occultation (COSMIC C001.2007.100.00.29.G05, where COSMIC is the Constellation Observing System for Meteorology Ionosphere and Climate: see Rocken et al., 2000 for system description), as given by its onboard GPS receiver. The signal is clearly detected during approximately $80 \mathrm{~s}$. Then the SNR for $\mathrm{L} 1$ and $\mathrm{L} 2$ become almost zero.

To realize the radiohologram power spectrum derived from a GPS occultation, $A(t)$ and carrier phase measurements are combined into a complex electric field $E(t)$, expressed as follows:

$E(t)=A(t) e^{i \varphi(t)}$

We will also construct a reference electric field (see Cardellach et al., 2004 for more details):

$E_{\mathrm{F}}(t)=A_{\mathrm{F}}(t) e^{i \varphi_{\mathrm{F}}(t)}$

We choose the amplitude and phase of this reference field to our convenience. The measured field can then be expressed relative to this reference:

$E(t)=B(t) \cdot E_{\mathrm{F}}(t)=A(t) / A_{\mathrm{F}}(t) e^{i\left(\varphi(t)-\varphi_{\mathrm{F}}(t)\right)} \cdot E_{\mathrm{F}}(t)$

If the reference field $E_{\mathrm{F}}(t)$ is judiciously chosen, the measured field can be expressed with a slowly varying complex (beating) function $B(t)$. Once the behavior with larger frequency has been separated, a frequency analysis of $B(t)$ will 


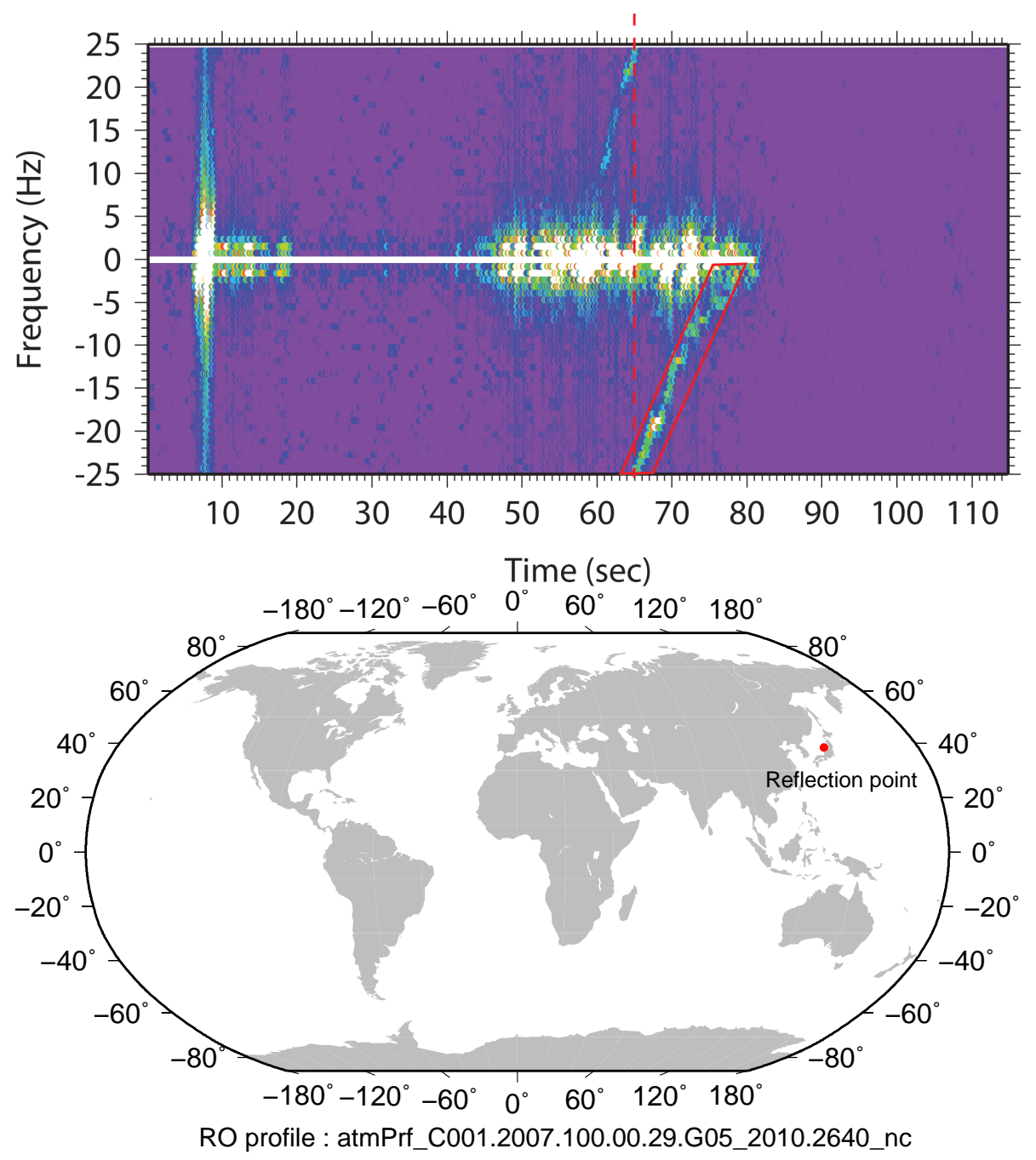

Fig. 2. (a) Temporal evolution of radio hologram power spectrum derived from GPS occultation on 10 April 2007 at 00:29 h (COSMIC-1 data file: atmPhs_C001.2007.100.00.29.G05_2007.3200). The red parallelogram represents the complementary mask that has been formed ad hoc to detect the reflected part of the signal. The mask is centered at $65 \mathrm{~s}$ with a slope of 0.52; (b) Geographical location of the GPS-RO event on 10 April 2007 at 00:29h.

show how $E(t)$ departs from the reference field, hopefully containing only low-frequency components. We will call radiohologram to a sliding Fourier Transform (FT) analysis of $B(t)$. This can help describing the internal structure of the signal, and identifying several close but distinct subcarriers within the signal. Depending on the circumstances, the existence of these subcarriers may be associated with the presence of multipath, the vertical structure of the propagation beam, or the existence of a reflection (Melbourne, 2004).

Since, in the case of COSMIC receivers, the sampling is done at $50 \mathrm{~Hz}$, this Fourier analysis can only explore a bandwith of $50 \mathrm{~Hz}$ around the reference field. It can therefore only be useful if the reference signal has been tuned to the target of study to better than $50 \mathrm{~Hz}$. The GRAS receiver can sample and record at $1 \mathrm{kHz}$, and this procedure could explore a bandwith of $1 \mathrm{kHz}$ around the reference field. Figure 2 (top side) shows the temporal evolution (x-axis) of the hologram's power spectrum of a COSMIC occultation. The vertical axis represents the shift in frequency with respect to the reference field. The geographical location of the reflection is also shown (bottom side). Time is measured since the beginning of the occultation. The reference field was chosen to mimic the main frequency component of the direct signal $\left(E_{\mathrm{F}}(t)\right.$ is a sliding average of $E(t))$. Since the power of the direct signal is substantially larger, it dominates the smoothed function. Thus the direct signal appears in the hologram with nearly zero frequency, broadened by atmospheric and ionospheric small-scale structure.

During most of the occultation, the power received at frequencies far from this broadened but clear direct signal has no structure evidently different from noise. Close to the surface touchdown of the direct signal, another component 
becomes evident. It is initially strongly aliased (much more than $25 \mathrm{~Hz}$ away from the direct signal), and also showing a large phase acceleration (its frequency changes). Both the phase acceleration and the frequency offset reduce, until this component finally merges with the direct signal. It is possible to identify that this component is associated with a reflection, rather than some other form of multipath, by the agreement of the frequency behavior with respect to the theoretical behavior of a reflection (e.g. Beyerle and Hocke, 2001).

The radio holographic analysis, as a means to separate direct and reflected signal components is also discussed in Pavelyev et al. (1996) and Hocke et al. (1999). Once identified that the component is a reflection, detailed analysis of the frequency shifts between direct and reflected signals can help determining atmospheric or surface properties. Complex patterns found in radio hologram spectra with a subset of observations at low latitudes are also been interpreted in terms of multipath propagation caused by layered structures in the refractivity field (Beyerle et al., 2002). Beyerle and Hocke (2001) also shows that GPS signals observed by the GPS/MET radio occultation experiment contain reflected signal components.

The frequency of the reference field is nearly that of the direct signal. The reflected signal has a substantial frequency shift in a large portion of the graph, several times larger than the bandwidth of $50 \mathrm{~Hz}$. It is also worth mentioning that since the receiver is tracking the direct signal, and not the reflected one, this latter is detected, and produces the interference, only because it also matches the C/A pattern of the direct signal.

There is no correlator in the receiver dedicated to track the reflected signal, and we depend on this interference to extract the properties of the reflection. This match in C/A code, however, occurs only if the relative delay between direct and reflected signals is smaller than 1 C/A chip, or $300 \mathrm{~m}$ (Parkinson and Spilker, 1996). The mismatch in optical lengths between both signals causes the reflected signal to be suboptimally detected, to only a fraction of its actual power, but still substantial. However, at $300 \mathrm{~m}$ or more of delay, the correlator that tracks the direct signal damps the reflected one by at least $20 \mathrm{~dB}$. Therefore, the reflected signal can only be extracted with the procedure mentioned if the optical path of both is less than $300 \mathrm{~m}$ apart. In Fig. 3, a raytracing estimation (see below, for more details of the raytracer) of the direct and reflected excess paths is shown. It can be seen that both signals are less than $1 \mathrm{C} / \mathrm{A}$ chip apart only during a fraction of the occultation, of the order of $20 \mathrm{~s}$. It is therefore only during this time window that we can expect to extract data describing the reflected signal, and therefore any signature that may be imprinted in it of the properties of the atmosphere.

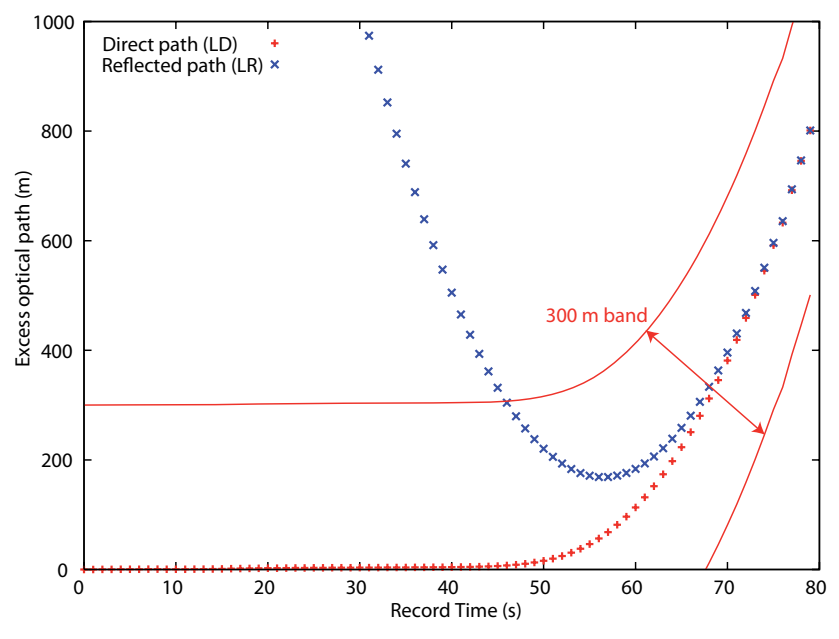

Fig. 3. Direct and reflected excess optical paths (m). The $300 \mathrm{~m}$ band represents the range of delays when the reflected signal will be captured by the correlator that tracks the direct signal, and interfere with it.

\section{Observables: the wrapped and unwrapped phases}

Given a beating function $B(t)=E(t) / E_{\mathrm{F}}(t)$, the hologram is its sliding Fourier Transform (FT):

$H(t, f)=\mathcal{F}[B(t)]$

In this case, it is interpreted that the direct signal is the power found at low frequency in the hologram, very close to the reference model, and the reflected signal, as the slant component, that appears aliased several times. We then select each of them applying some hologram mask $D$, that selects the direct signal, and a complementary mask $R=I-D$, where $I$ is the identity. Since the objective of this study is to explore the geophysical content of the reflection, rather than a generic algorithmic definition of this mask, we have here selected $R$ (and its complement $D$ ) conservatively, with an ad hoc definition of the slant area of the hologram. This should be refined into a more generic, and especially automatic, definition. The two signals appear separated by a clear region in frequency which contains very low power. Therefore the exact shape of the mask is not critical, as long as it separates the two clearly defined signals.

For the occultation profile studied here (COSMIC's C001.2007.100.00.29.G05) the reflection begins to be detectable around $t=65 \mathrm{~s}$. The mask $R$ is applied beginning at this time and with an orientation of its approximate phase acceleration $\left(0.52 \mathrm{~Hz} \mathrm{~s}^{-1}\right)$. The mask is marked in the hologram with a red parallelogram (Fig. 2). The exact definition of this boundary is therefore not critical. The separation cannot of course be carried out perfectly, as the frequency of both signals is too close near their merging (around $75 \mathrm{~s}$ ), or is aliased (e.g. around $60 \mathrm{~s}$ ) into the direct signal. The only location where the mask is difficult to place, and subject to an arbitrary definition, is the upper boundary 

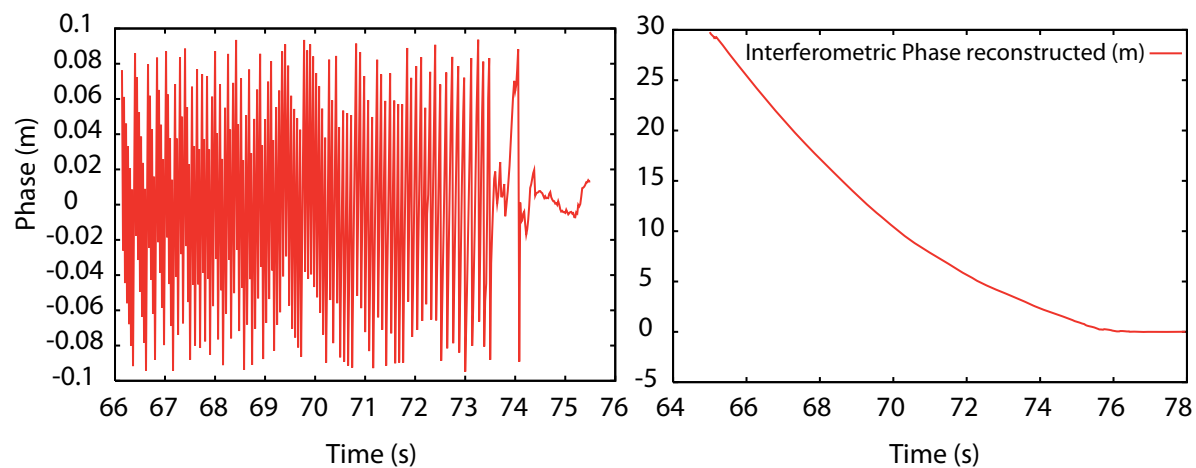

Fig. 4. Phase difference between the reference signal and the reflected signal (left). This phase difference can also be unwrapped removing the $2 \pi$ jumps to get the interferometric phase (right).

of the parallelogram, near zero frequency, because the two signals merge. However, at zero frequency separation, the two signals do not turn with respect to each other, and do not accumulate a relative phase, which is the observable here. On the other hand, a receiver with higher sampling (such as GRAS) could separate better the two propagation channels.

Reverting with an inverse FT to the beating function space, the masks define two beating functions:

$$
B_{\mathrm{D}}(t)=\mathcal{F}^{-1} D[H(t, f)]
$$

and

$$
B_{\mathrm{R}}(t)=\mathcal{F}^{-1} R[H(t, f)]
$$

which we will identify as the beating functions of the separated direct and reflected signals. This eventually allows the reconstruction of the two components of the electromagnetic field.

It is here evident that a judicious choice of the reference field is critical, as otherwise no practical mask will separate the two components properly. This sliding mean is a reasonable choice, as the direct signal is substantially stronger than the reflected, dominating the mean and leaving the direct signal into a simple geometry: a straight line around zero frequency, with moderate broadening. We must mention that this clear case is very frequent, and there was no particular effort of selection of a "good" case, other than the selection of a reflection over ocean, to fix the vertical location of the reflecting surface. For a fraction of cases, the two signals are not as clearly separable. However, our focus is to explore those clear cases, to determine whether there is valuable information currently not being used, before exploring how to handle the difficult cases.

Each of the two beating functions $B_{\mathrm{D}}(t)$ and $B_{\mathrm{R}}(t)$ represents a slowly rotating phasor around the reference field $E_{\mathrm{F}}(t)$. The phasors are known only modulo $2 \pi$, but could be unwrapped (reconnected) to continuous functions removing the jumps of $2 \pi$. The signal represented by $B_{\mathrm{D}}(t)$ can be identified during most of the recording period, from 0 to $80 \mathrm{~s}$. The phasor $B_{\mathrm{D}}(t)$ is very similar to the beating function from the full recorded signal, as only a small amount of power has been removed. It is however free from most of the interference of the surface. In any case, we assume that the processing and interpretation of this component are standard practice, and we will not discuss it further.

The signal $B_{\mathrm{R}}(t)$ has a significant amplitude only during a small portion of the occultation ( $55 \mathrm{~s}$ to $80 \mathrm{~s}$ ). Regardless of the exact mask chosen, this could be expected for any reasonable mask, since at earlier times the reflected signal was outside the C/A chip of the direct signal, and was ignored by the correlator. It is also a slow rotating phasor (slow compared to $\left.E_{\mathrm{R}}(t)\right)$, although faster than $B_{\mathrm{D}}(t)$, since the reference field is not tuned to follow this signal. It can also be reconnected, removing the $2 \pi$ jumps, to form another signal, that we will interpret as the reflected signal.

The delays between the reconnected direct, reconnected reflected and reference signals, allows us to retrieve the equivalent excess of phase between any pair of them. On the left side, Fig. 4 shows the excess of phase obtained between the reflected signal and the reference one, that is, the phase of $B_{\mathrm{R}}(t)$. We call this phasor the interferometric signal between both. The oscillations indicate the existence of a relative rotation between the two signals by several turns (it is wrapped). The plot on the right side represents the same signal, with all $2 \pi$ jumps removed. We will call this the unwrapped interferometric signal, equivalent to the relative delay between the reflected and the direct ray path, and it will be our primary observable for the extraction of atmospheric properties.

\section{Ray tracing analysis}

Ray tracing is a numerical procedure that explicitly evaluates the propagation trajectories of the electromagnetic waves. In this study we use ray tracing with the purpose of determining not only the optical paths, but also the trajectories, which indicate the regions of the atmosphere that are being probed by a given propagation beam. The ray tracing is computed between an emission point (transmitter, in general a GNSS satellite orbiting at heights of about $20000 \mathrm{~km}$ ) and a receiver point (here, a LEO satellite). 
Wave propagation calculation can be done in two ways. The first is the geometrical approach. Only the direction of propagation of electromagnetic is used. Signal propagation is thus reduced to the knowledge of the rays' characteristics and signal wave lengths. Signal properties are influenced by the change of refractivity across the material medium. In the second method, signal propagation can be represented as an ondulatory model, which is computationally more complex. In the case of GPS radio occultation, the wave character of light is not negligible, but small: signal propagates as a beam of non-negligible width, of the order of the Fresnel diameter. Although not negligibly so, the Fresnel diameter is moderately small with respect to other cross-beam length scales, such as the vertical structure of the atmosphere.

However, for our main purpose, which is the identification of the interaction between the beams and each layer of the atmosphere, this width is not critical, and we will neglect the wave nature of GPS signals in this first test. We will therefore describe light waves as propagating in a direction orthogonal to the geometrical wavefronts defined as the surfaces on which the signal phase is constant.

\subsection{Ray path determination}

The index of refraction, $n$ in some medium is defined as the speed of light in vacuum divided by the speed of light in the medium. In the atmosphere, the index of refraction is very close to unity. Consequently, it is more common to express it in terms of refractivity $N$. By definition, $N=(n-1) \times 10^{6}$. Our final objective is to extract the unknown field of refraction index $n(\boldsymbol{x})$ from some form of measurement, or combination, of the optical lengths $L_{\mathrm{obs}}$ through different propagation paths.

The total optical length $L$ of a given path $p$, can be expressed as:

$$
L=\int_{\mathrm{p}} n(\boldsymbol{x}) d s
$$

with $n(\boldsymbol{x})$ defined as the refraction index and $s$ the geometric position along the path. Let us choose an initial field $n_{0}(\boldsymbol{x})$. This initial field is used as a reference, and may or may not be vacuum. The corresponding total optical length would be $L_{0}$. This will differ from $L$ due both to a geometrically different path $p_{0}$, and a different speed of light along the path. However, $n_{0}(\boldsymbol{x})$ is known, which allows us to evaluate trajectories numerically over it, as well as other properties.

Given some observations, the differences $L_{\mathrm{obs}}-L_{0}$ for each ray contain some information concerning the errors in having assumed the field $n_{0}(\boldsymbol{x})$ as an approximation to $n(\boldsymbol{x})$. Given an array of many different configurations, and thus many trajectories over the same field, this allows to perform some kind of tomographic reconstruction of the field. This tomographic approach was taken by Flores et al. (2001), under the assumption of straight line propagation, equivalent to use a constant field, such as vacuum, as the reference field $n_{0}(\boldsymbol{x})$. Alternatively, we may also interpret it as an assumption that, even if the field is not zero, that only the difference in speed is relevant, whereas the geometric difference of the paths through the fields $n_{0}$ and $n$ would be negligible.

In this study, we consider instead geometrical configurations where the bending of the ray is relevant and non-zero, but small (around $1^{\circ}$ ). A substantial fraction of the excess optical path is therefore geometric, since the trajectory is not a straight line, the rest deriving from the propagation speed along the trajectory. From the two reasons why the optical lengths $L$ and $L_{0}$ differ (geometric length and propagation speed) we will assume that we have an initial field $n_{0}$ is sufficiently close to the actual one $n$, so that the error in the length of the trajectory is indeed negligible. Indeed, since optical trajectories are stationary with respect to the propagation paths, a geometrical error in the trajectory is only of second order.

This means that we can evaluate the optical length of stationary trajectories over fields other than the known $n_{0}$, without having to evaluate the geometric variation of the trajectory, as long as we do not exceed the linear regime. This allows us to search for the true field $n$ in the (hugely dimensional) space of possible refractivity fields, having evaluated the geometrical shape in only one of these fields, i.e. $n_{0}$.

\subsection{Variation of the optical path length and refractivity field}

The quantity we want to obtain is the inaccuracy $\varepsilon_{n}(\boldsymbol{x})$ of the guessed refraction index. The inaccuracy of the optical path of a given ray can be expressed to first order as:

$$
\delta L=\frac{\delta L}{\delta n(\boldsymbol{x})} \delta n(\boldsymbol{x})+\frac{\delta L}{\delta p} \frac{\delta p[n]}{\delta n(\boldsymbol{x})} \delta n(\boldsymbol{x})
$$

The first term expresses the speed term of the optical path, the second one the geometric dependence. Due to stationary trajectory the second term vanishes. The above expression reduces to:

$\varepsilon_{\mathrm{L}}=\frac{\delta L}{\delta n(\boldsymbol{x})} \varepsilon_{n}(\boldsymbol{x})$

The solution to this functional equation also follows an associated Euler set of differential equations (Gelfand and Fomin, 2000; Born and Wolf, 1980). The systems of differential equations and boundary conditions are summarized as:

Differential equations $\left(S_{1}\right):\left\{\begin{array}{l}\frac{d^{2} x_{i}}{d s^{2}}=\frac{1}{n}\left[\frac{\delta n}{\delta x_{i}}-\frac{d n}{d s} \frac{d x_{i}}{d s}\right] \\ \frac{d L}{d s}=n\end{array}\right.$

where $s$ is the geometrical path along the trajectory.

Boundary conditions $\left(S_{2}\right):\left\{\begin{array}{l}x_{0}=x_{\mathrm{GPS}} \\ x_{\mathrm{F}}=x_{\mathrm{LEO}} \\ L_{0}=0\end{array}\right.$

0 and $F$ express the initial and final points of the trajectory. 

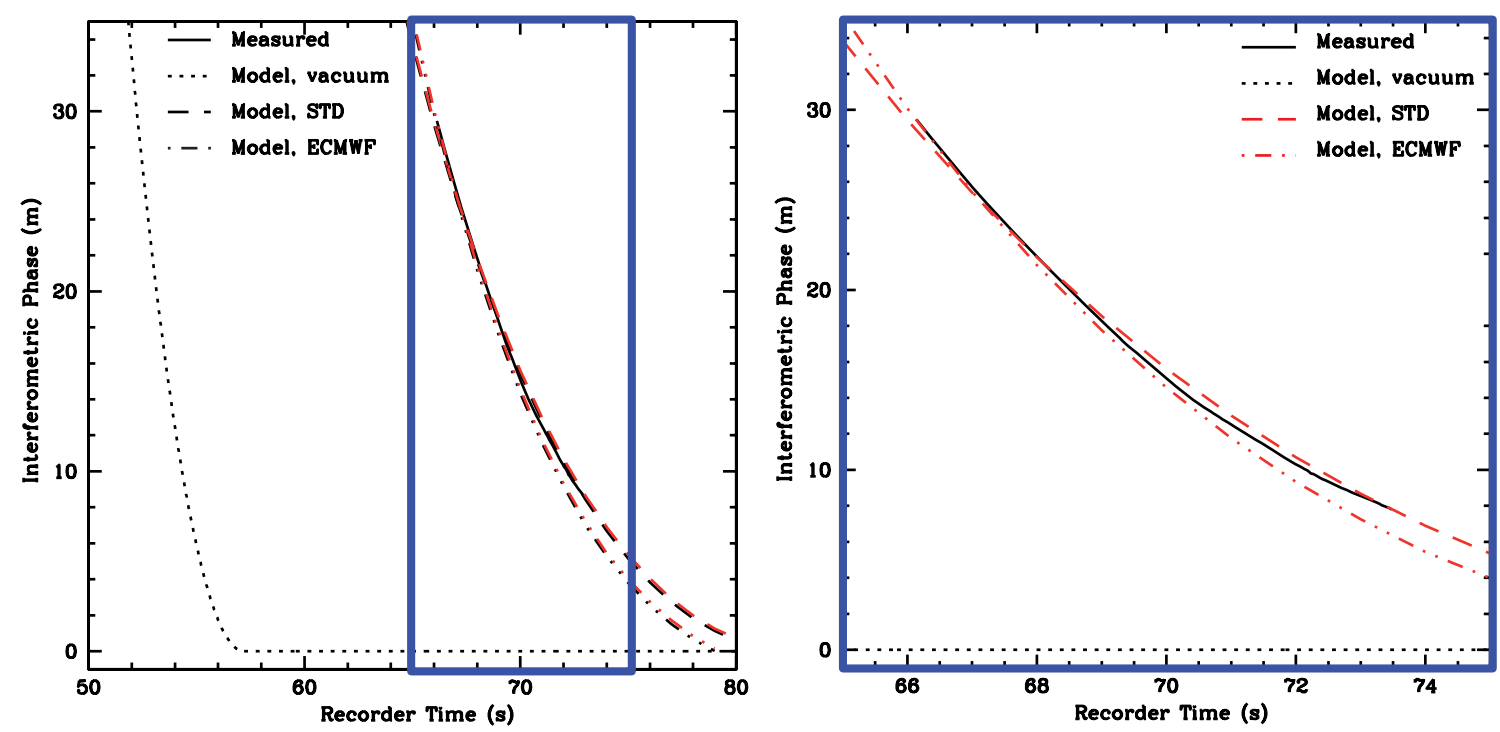

Fig. 5. Path difference between direct and reflected signals over sea using interferometric phases measured and evaluated with raytracing. Black evaluated curves do not use ionosphere correction. Red evaluated curves use COSMIC's estimation. On right hand side a zoom of the same path difference is shown.

The solution to the system of differential equations $S_{1}$ delivers the ray trajectory and the optical path length. In order to realize the inversion and retrieve the refraction index field, we must extract from the obtained ray trajectories the regions of the atmosphere that are participating in the propagation of every ray, and compare the observed and predicted delays.

\subsection{Ray tracing with COSMIC data}

In this study we use COSMIC data. The atmospheric and ionospheric profiles are available from the radio occultation itself, as well as ECMWF guess fields. The raytracer can resolve the trajectories of the rays over the available guess fields. We represent here both direct and reflected path lengths along an occultation event (Fig. 3). We plot the $300 \mathrm{~m}$ band around the different signals to show the available information received from the GPS correlator. The new information brought by the reflected signal is depicted between 45 to $65 \mathrm{~s}$.

Next, a comparison on the difference between path calculated from real data ("measured") and evaluated by the ray tracing is done. The "measured" one corresponds to the interferometric unwrapped phase. The evaluated path length is estimated over the following five guess fields:

1. The use of an exponential atmosphere (labeled STD) that is a simple model $N=300 \exp ^{\frac{-h}{H}}$, with $H=\frac{15 \mathrm{~km}}{\log (10)}$, without ionosphere.

2. The exponential atmosphere above (STD), but adding a ionosphere as given by the COSMIC estimation.

3. ECMWF guess field, without ionosphere.
4. ECMWF guess field, with ionosphere as extracted by COSMIC.

5. A reference vacuum ray tracing (straight lines).

Whenever the ionosphere is present, the ionospheric refractive index is evaluated for the L1 frequency, as only the L1 interferometric phase had been extracted. The comparison is shown on Fig. 5.

\section{Inversion procedure to retrieve refractivity field}

In the following we show the result of inversion procedures to obtain a solution refractivity field, with the use of data generated by the raytracing procedure itself. The raytracing may allow in principle to analyze 3-D fields. However a full 3-D solution would require massive amounts of constraining data, which generally will not be available. We restrict, then, to the case of a quasi-spherical solution, which is only a function of the altitude over the mean sea level. To realize the inversion procedure that leads to the refractivity field we explore here two possibilities.

One is a perturbative approach. We specify a number of vertical layers, and we assume that the 3-D solution field has the same shape as the guess field, but differs from it by a small factor in each of the vertical layers. We are therefore searching for an optimal solution within a multidimensional space of possible refractivity fields. This space has as many dimensions as layers we have defined where the solution field can depart from the guess. 
During raytracing, we can collect geometric information of the trajectory. This allows the evaluation of $\frac{\delta L}{\delta n_{i}}$, for each layer $i$, and therefore the dependence of each ray on each of the defined layers. The number and size of the layers should of course be chosen judiciously, so that the solution is indeed constrained by the data. The guess field is a member of this multidimensional space: the one which does not have any departure. The raytracing, and its associated geometric information is needed for only one refractivity field, and we choose the guess field for it. The neighbourhood is only described to the linear level of departure from the guess field. The solution is selected through a least squares procedure, whose details are described in the following section.

We also consider another approach, that follows Cardellach et al. (2004), and that serves to illustrate the perturbative approach, of which it is a special case. Following the perturbative approach, we define only one single layer, where the solution field departs from the guess field by a constant factor, which is the same everywhere. The guess field is the case where this constant factor is 1 . Since the defined solution space is small, 1-D, we can afford to launch a raytracing over several fields of this space, including the guess field and some other fields in its neighbourhood.

In all cases this explores the potential sensitivity of a reflection observable to detect the variations in tropospheric parameters. The experiment has been run defining the reflection-observable as the carrier-phase delay between the reflected radio-link and the direct one, which has a formal precision of the order of few-centimeters. The ray tracer detailed previously is used to track both direct and reflected signals. An ECMWF refractivity profile is used as the guess field. In all state-of-the-art NWP systems, the short-term forecast error of refractivity in the low troposphere is in the range $1 \%-10 \%$, which justifies exploring the solution space at each $1 \%$ around a short-term forecast. In the 1-D approach, the defined solution space is sampled with several guess fields modified by applying a constant factor to the ECMWF field, this factor being 0.98, 0.99, 1.00, 1.01, and 1.02 .

For a $1 \%$ variation on refractivity, a relative change on humidity ranging from 0.2 to $0.5 \mathrm{~g} \mathrm{~kg}^{-1}$ is expected. The mean temperature equivalent error would be around $0.5 \mathrm{~K}$.

Then the corresponding simulated reflection-observable is generated. In total several profiles are obtained, and are shown in Fig. 6. The figure also shows the interferometric phase from the reflection that appears in COSMIC occultation C001 2007.100.00.29.G05. The $1 \%$ variation introduces between 0.2 to $0.75 \mathrm{~m}$ variations in the carrier-phase delay, which is an order of magnitude larger than the formal error of the observables (see errorbars in the in-set of Fig. 6). Although this academic exercise does not take into account the variation of refractivity from one layer to another, it does show that the precision of the measure is sufficient to contain useful information that, translated to refractivity, is comparable to approximately $0.1 \%$ in the low troposphere.

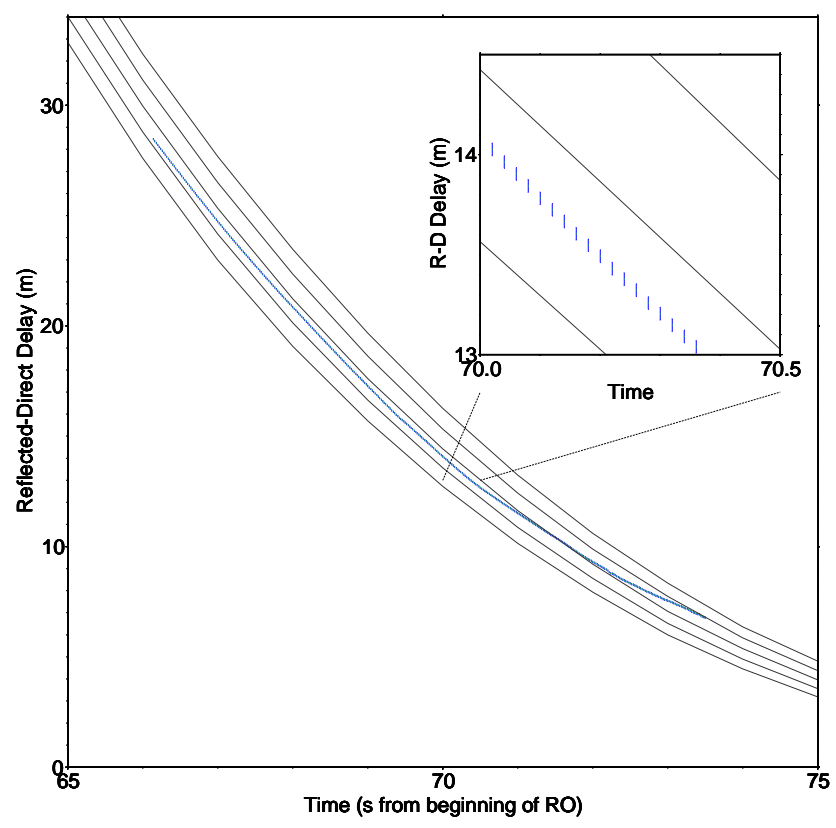

Fig. 6. Comparison between interferometric delay (observables with error bars) and the ray tracer simulated observables, based on the ECMWF atmospheric profile. A 1-D family of profiles is explored, varying the refractivity in steps of $1 \%$. From left to right, the curves represent the raytracing over $0.98,0.99,1.00,1.01$ and 1.02 times the ECMWF profile.

\subsection{Least squares method and inversion procedure}

From the obtained ray trajectories issued from the so-called OAT ray-tracer model (Aparicio and Rius, 2004) prepared to track both direct and reflected signals, we must determine which regions of the atmosphere divided in finite elements are participating in the propagation of every ray. We then compare the expected and observed delays. The ray tracing delivers a prediction for the observable delay $L^{0}$, that can be decomposed as follows:

$L^{0}=l^{0}+g^{0}+a^{0}$

with $l^{0}$ the straight line delay, $g^{0}$ the excess geometric delay due to ray bending and $a^{0}$ the atmospheric delay. We can calculate with very high accuracy the delay $l^{0}$, thanks to the positions of the emitter and receiver.

If we decompose the reference atmospheric field $n_{0}$ into finite elements, which we will assume as a number of quasispherical shells or layers, bounded by surfaces of constant altitude over the mean sea level, the analysis of the ray tracing can also deliver the linear contribution of each finite element to $L^{0}$. This is the optical length $\int\left[n_{0}(\boldsymbol{x})-1\right] d s$ accumulated along the trajectory within the layer.

Then, for every ray, we can assign a weight $w$ to each finite element proportional to its contribution to the excess optical length. The result is an ensemble $w_{\mathrm{r} j}$, that is, the exess optical length to ray $r$ that accumulated while it was propagating 
into the finite element $j$. In the case of a path difference, as in the interferometric phases, the weights are the differences between the direct and reflected trajectories. If we have measurements $L_{\mathrm{r}}^{\text {obs }}$ of the path delays $L_{\mathrm{r}}$, we can use a minimization approach to obtain the most probable error field in our assumed refractivity. To describe these errors we express a list of unknown relative errors $\epsilon_{j}$ in our knowledge of the refractivity in each finite element. We get:

$N_{j}=N_{j}^{0}\left(1+\varepsilon_{j}\right)$

The difference between the reference and actual path delays can be expressed as follows:

$L_{\mathrm{r}}-\left(l_{\mathrm{r}}^{0}+g_{\mathrm{r}}^{0}\right)=\sum_{j} w_{\mathrm{r} j}\left(1+\varepsilon_{j}\right)$

Then the observationnal measurement taking into account the observationnal error $\epsilon_{\mathrm{r}}$ becomes:

$L_{\mathrm{r}}^{\mathrm{obs}}-\left(l_{\mathrm{r}}^{0}+g_{\mathrm{r}}^{0}\right)=\sum_{j} w_{\mathrm{r} j}\left(1+\varepsilon_{j}\right)+\epsilon_{\mathrm{r}}$

This expression can be expressed in a matrix form as:

$\overline{\Delta L}=\bar{W} \varepsilon+\bar{E}$

To solve the Eq. (16) optimally we use a least squares method. The ensemble $E$ of measurement errors is not known accurately, but we will assume that it is small and constant. The phases can be extracted with a precision of few centimeters, and the analysis of the 1-D case indicates that this is small compared with the optical length involved.

Therefore we solve the matrix expression as $\overline{\Delta L}=\bar{W} \varepsilon$. Consequently, the solution to $\varepsilon$ contains some errors caused by having neglected the measurement errors, as well as model errors. Model errors can be related to the assumption that the finite layers were sufficiently small, the geometric optics, atmospheric and ionospheric scintillation, or in position errors of the emitter or the receiver. We will here assume that both model and position errors are small. To find the least squares solution of Eq. (16), we construct the system of normal equations, as explained in Golub (1965): $\bar{W}^{T} \overline{\Delta L}=\bar{W}^{T} \bar{W} \varepsilon$. This sytem of equations can be solved to:

$\varepsilon=\left(\bar{W}^{T} \bar{W}\right)^{-1} \bar{W}^{T} \overline{\Delta L}$

Thus, we get the most probable solution $\varepsilon$ as a least squares solution and also the corresponding covariance matrix $\mathbf{C}=s_{0}^{2}\left(\bar{W}^{T} \bar{W}\right)^{-1}$. This solution can be interpreted as the fractional deviations of the solution refractivity field with respect to the reference field. The scale factor $s_{0}^{2}$ is equal to $v^{T} \boldsymbol{v} / f$, with $\boldsymbol{v}$ the vector of residuals, and $f$ the number of degrees of freedom of the fit. Therefore, the ray tracing has been used to record the contribution of each finite element to each ray. The least-squares procedure delivers the most probable error field of the guess and the covariance matrix of the result.

\subsection{Selection of observables for the inversion}

In the former section, we have considered some hypothetical observables of optical path, which may have been gathered over an array of paths. Actual observables are not directly any optical path, but rather combinations of several optical paths, along several trajectories. In particular, we have extracted unwrapped phases over the direct and reflected paths. These phases are not optical paths, but differences between optical paths. However, this does not change the least squares procedure of inversion, as these are linear combinations of the system of equations $\overline{\Delta L}=\bar{W} \varepsilon$

The original system is based on the output of the raytracing. We may simply combine linearly this system according to the observables that we actually have. In addition, given that we are analyzing the reflected signal, and that we have extracted it through its interference with the direct signal, therefore essentially extracting the relative phase between both: our observable will be the difference between the optical lengths through the direct and reflected propagation paths. This difference is of course a linear combination of two of the raytracings.

We then have a ray tracing function, such as: $f(n)=L$ Then, applying the derivative we get:

$f(n)=f_{0}\left(n_{0}\right)+W\left(n-n_{0}\right)$

with $n_{0}$ as a reference field, and $W$ is the ensemble of dependences, which, as shown above, depend only on the optical excess lengths, but not on the geometric difference of the trajectory. An equation of the following kind, expresses the relationship between the error of the field, and observables: $W \Delta n=\Delta \varphi$

Where $W$ stems from the raytracing. Then, the least squares method is used to solve the equation and the final equation to solve becomes:

$\Delta n=\left(W^{T} W\right)^{-1} W^{T} \Delta \varphi$

The solution allows a series of independant layers with different refractivity index (Fig. 7). The least squares procedure, described above, finds the best solution within this space. The profile is not shown below $1 \mathrm{~km}$ since the solution is not well constrained by data. As a consequence the refraction index correction is not realistic. We therefore show only the results whose solution was well constrained. We remind that here the objective of the study is not to show that a full profile can be derived from reflection data, but that there is an information that can provide useful data of at least a portion of the profile. 


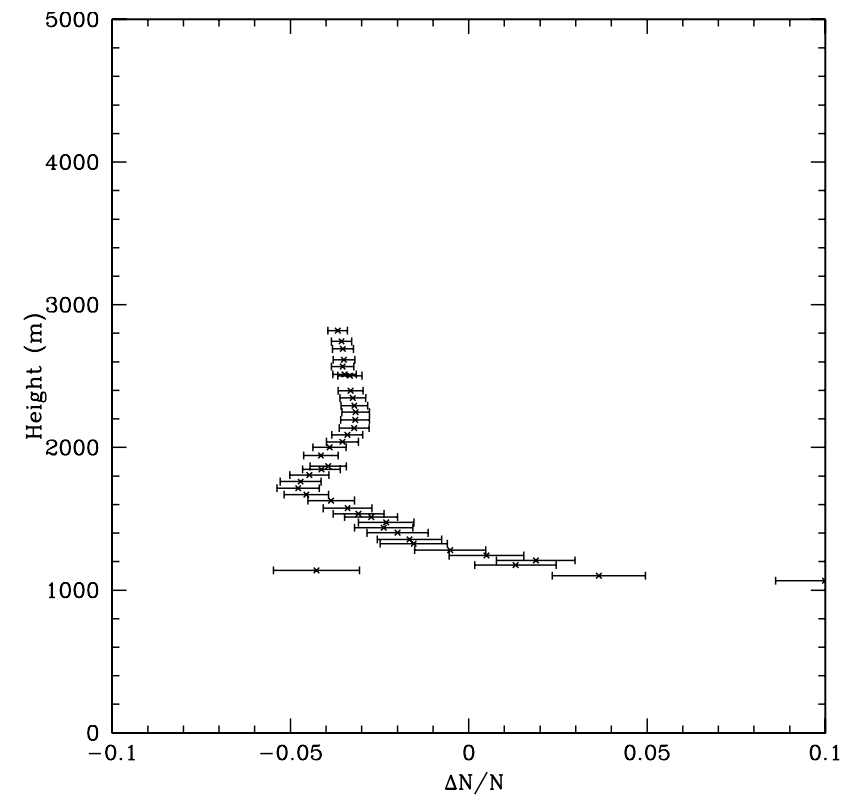

Fig. 7. Refractivity correction coefficients $\left(\frac{\Delta N}{N}\right)$ to the ECMWF profile. A multidimensional solution space has provided this solution. It allows a serie of independant layers with different refractivity index respectively.

\section{Conclusions}

We can see that the interferometric unwrapped phase is very close to the optical path difference between direct and reflected trajectories, as evaluated over a numerical guess field. The fields that are likely to be of superior quality (ECMWF, ionosphere included), are $x$ closer. Furthermore, the precision of the interferometric observables is an order of magnitude smaller than changing by $1 \%$ the atmospheric profiles. Firstly, this supports that the interferometric unwrapped phase is a potentially useful observable, that is recoverable and can be potentially used. The observable is quantitatively extracted with high accuracy, supporting its potential.

Secondly, the raytracing allows the interpretation of this observable data, and provides the elements to choose a solution within the space of refraction index fields neighbouring the guess field. A search in a layered space of 20 dimensions (i.e. there were 20 low tropospheric layers, each allowed to vary independently) showed that the level of constrain is sufficient to be useful.

This study was a first attempt to evaluate the reflected signals potentiality in terms of tropospheric information content. In this paper we introduced the way the reflected signals occuring during an occultation event could be extracted from the direct one. Signal separation was conducted using radio-holographic analysis. Then the spectral content of each signal was interpreted computing the direct and reflected phases. Then the relative delay between the reconnected direct and reflected signals was extracted. The ray tracing procedure allowed the interpretation and inversion of the relative delay to a refractivity field through a perturbative approach. The ray-tracer model allows to track both direct and reflected signals. As demonstrated in the sensitivity study made in Sect. 5, tropospheric information content is embedded in the RO reflected signals. In addition, we have shown that reflection-observables are able to capture variations in the tropospheric profile. This ability to sense and refine the variations in the troposphere would be of great interest to improve the tropospheric information content in near-surface.

We have shown that it is possible to do realistic ray tracing comparison between measured unwrapped phase and over realistic refractivity models. The comparison has been done taking into account the ionospheric state. The computation of the non-vacuum ray tracing methods have shown to be reasonably close to the unwrapped reflected phase. Also, the least squares inversion allows the extraction of useful meteorological information. We highlight the potential of GPS-RO reflected signals to sense the lowermost layers of the troposphere. The main objective of the work consists in determining the ability of such a measurement to quantify the properties of the low atmosphere (refractivity profile within the boundary layer). The perturbation procedure presented here is in principle applicable to any profile. Nevertheless, given the cost of the raytracing, we consider it practical only if the perturbation inversion is sufficiently accurate after one iteration of raytracing, perturbation and inversion. This of course constrains to cases where the a priori information is already close to the solution. Strong gradients presumably reduce the convergence rate for these inversions. However, this may still be beneficial as the reflected signal may probe the gradient at a different angle as the direct signal, providing information difficult to extract from the direct signal. A full assesment, however, requires merging both sources of data, which was beyond the scope, and is still underway.

Acknowledgements. Funding to develop the GPS-RO reflected signals retrieval was provided by the Canadian Space Agency, EUMETSAT and the GRAS SAF. K. B. benefits from a NSERC (Natural Siences and Engineering Research Council of Canada) postdoctoral fellowship. E. C. is a Spanish "Ramón y Cajal" awardee.

Edited by: U. Foelsche

\section{References}

Ao, C., Meehan, T., Hajj, G., Mannucci, A. J., and Beyerle, G.: Lower troposphere refractivity bias in GPS occultation retrievals, J. Geophys. Res., 108, 4577, 2003.

Aparicio, J. M. and Deblonde, G.: Impact of the Assimilation of CHAMP Refractivity Profiles on Environment Canada Global Forecasts, Mon. Weather Rev., 136, 257-275, doi:10.1175/2007MWR1951.1, 2008. 
Aparicio, J. M. and Rius, A.: A raytracing inversion procedure for the extraction of the atmospheric refractivity from GNSS travel-time data, Parts A/B/C, probing the Atmosphere with Geodetic Techniques, Phys. Chem. Earth, 29, 213-224, doi:10.1016/j.pce.2004.01.008, 2004.

Beyerle, G. and Hocke, K.: Observation and simulation of direct and reflected GPS signals in Radio Occultation Experiments, Geophys. Res. Lett., 28, 1895-1898, 2001.

Beyerle, G., Hocke, K., Wickert, J., Schmidt, T., Marquardt, C., and Reigber, C.: GPS radio occultations with CHAMP: A radio holographic analysis of GPS signal propagation in the troposphere and surface reflections, J. Geophys. Res., 107(D24), 4802, doi:10.1029/2001JD001402, 2002.

Born, M. and Wolf, E.: Principles of optics: Electromagnetic theory of propagation, interference and diffraction of light, Pergamon Press, London, 1980.

Cardellach, E., Ao, C. O., de la Torre Juárez, M., and Hajj, G.: Carrier phase delay altimetry with GPS-reflection/occultation interferometry from low Earth orbiters, Geophy. Res. Lett., 31, L10402, doi:10.1029/2004GL019775, 2004.

Cardellach, E., Oliveras, S., and Rius, A.: Applications of the Reflected signals found in GNSS Radio Occultation Events, in: Proceedings of GRAS SAF Workshop on Applications of GPS radio occultation measurements, 133-143, 2008.

Cucurull, L., Kuo, Y.-H., Barker, D., and Rizvi, S. R. H.: Assessing the Impact of Simulated COSMIC GPS Radio Occultation Data on Weather Analysis over the Antarctic: A Case Study, Mon. Weather Rev., 134, 3283-3296, doi:10.1175/MWR3241.1, 2006.

Fjeldbo, G., Kliore, A. J., and Eshleman, V. R.: The neutral atmosphere of Venus as studied with the Mariner V radio occultation experiments, Astronom. J., 76, 123-140, 1971.

Flores, A., Rius, A., Vilà-Guerau de Arellano, J., and Escudero, A.: Spatio-Temporal Tomography of the Lower Troposphere Using GPS Signals, Phys. Chem. Earth, 26, 405-411, 2001.

Gelfand, I. and Fomin, S.: Calculus of Variations, Dover Publ., 2000.

Golub, G.: Numerical methods for solving linear least squares problems, Numer. Math., 7, 206-216, 1965.

Gorbunov, M.: Accuracy of the refractometric method in a horizontally nonuniform atmosphere, Izv., Atmos. Ocean. Phys., 5, 381-384, 1988.

Gurvich, A., Kan, V., Popov, L., Ryumin, V., Savchenko, S., and Sokolovskiy, S.: Reconstruction of the atmosphere's temperature profile from motion pictures of the Sun and Moon taken from the Salyut-6 orbiter, Izv., Atmos. Ocean. Phys., 1, 7-13, 1982.

Hajj, G., Ibanez-Meier, R., Kursinski, E., and Romans, L.: Imaging the ionosphere with the global positioning system, Int. J. Imag. Syst. Tech., 5, 174-184, 1994.
Healy, S.: Assimilation of GPS radio occultation measurements at ECMWF, in: Proceedings of GRAS SAF Workshop on Applications of GPS radio occcultations measurements, 99-109, 2008.

Hocke, K., Pavelyev, A. G., Yakovlev, O. I., Barthes, L., and Jakowski, N.: Radio occultation data analysis by the radioholographic method, J. Atmos. Sol.-Terr. Phy., 61, 1169-1177, doi:10.1016/S1364-6826(99)00080-2, 1999.

Kursinski, E. R., Hajj, G. A., Bertiger, W. I., Leroy, S. S., Meehan, T. K., Romans, L. J., Schofield, J. T., McCleese, D. J., Melbourne, W. G., Thornton, C. L., Yunck, T. P., Eyre, J. R., and Nagatani, R. N.: Initial Results of Radio Occultation Observations of Earth's Atmosphere Using the Global Positioning System, Science, 271, 1107-1110, doi:10.1126/science.271.5252.1107, 1996.

Melbourne, W. G.: Radio occultations using Earth satellites: A wave theory treatment, vol. Monograph 6 , jet propulsion laboratory california institute of technology edn., Wiley InterScience, 2004.

Parkinson, B. W. and Spilker, J. J.: Global Positioning System: Theory and Applications, vol. 1, American Institute of Aeronautics, 1996.

Pavelyev, A., Volkov, A., Zakharov, A., Krutikh, S., and Kucherjavenkov, A.: Bistatic radar as a tool for earth investigation using small satellites, IAAA International Symposium on Small Satellites for Earth Observation, Acta Astronaut., 39, 721-730, doi:10.1016/S0094-5765(97)00055-6, 1996.

Poli, P.: Preliminary assessment of the scalability of GPS radio occultations impact in numerical weather prediction, Geophys. Res. Lett., 35, L23811, doi:10.1029/2008GL035873, 2008.

Raymund, T., Austen, J., Franke, S., Liu, C., Klobuchar, J., and Stalker, J.: Application of computerized tomography to the investigation of ionospheric structures, Radio Sci., 25, 771-789, 1990.

Rennie, M. P.: The impact of GPS radio occultation assimilation at the Met Office, Q. J. Roy. Meteorol. Soc., 136, 116-131, doi:10.1002/qj.521, 2010.

Rocken, C., Kuo, Y.-H., Schreiner, W., Hunt, D., Sokolovskiy, S., and McCormick, C.: COSMIC System Description, Terr. Atmos. Ocean. Sci., 11, 21-52, 2000.

Sokolovskiy, S.: Solution of the inverse refraction problem by sensing of the atmosphere from the space, Sov. J. Remote Sens., 3, 333-338, 1990.

Sokolovskiy, S., Rocken, C., Schreiner, W., Hunt, D., and Johnson, J.: Postprocessing of L1 GPS radio occultation signals recorded in open-loop mode, Radio Sci., 44, RS2002, doi:10.1029/2008RS003907, 2009. 